\title{
Contrast enhanced ultrasound and magnetic resonance imaging in hepatocellular carcinoma diagnosis
}

\author{
Cristiana I. Dumitrescu', Ioana A. Gheonea ${ }^{2}$, Larisa Săndulescu ${ }^{3}$, Valeriu Surlin ${ }^{4}$, \\ Adrian Săftoiu ${ }^{3}$, Daniela Dumitrescu ${ }^{2}$
}

${ }^{1}$ Pharmacology Department, ${ }^{2}$ Radiology and Imaging Department, ${ }^{3}$ Gastroenterology Department, ${ }^{4}$ Surgery Department, University of Medicine and Pharmacy Craiova, Romania

\begin{abstract}
Background: The new developments in imaging technology, including contrast enhanced ultrasound (CEUS), computed tomography (CT), and magnetic resonance imaging (MRI), allow a better diagnosis of both malignant and benign liver lesions. Material and methods: A retrospective trial of 126 patients was conducted in the Gastroenterology and Imaging Departments of the University of Medicine and Pharmacy Craiova, Romania. CEUS and MRI were the imaging techniques used for diagnosis of focal liver lesions (FLL), especially for hepatocellular carcinoma (HCC). Histopathology was used only in 15 cases. For each method of investigation we calculated the sensitivity, specificity, positive and negative predictive values (PPV and NPV), positive and negative likelihood ratio (+LR, -LR), accuracy and we compared the ROC curves. Statistical analysis also included the Chi-square and Kappa tests. Results: Seventy six cases were diagnosed as HCC, with average size of $5.2 \pm 3.3 \mathrm{~cm}$ in diameter. The sensitivity and specificity were $71.4 \%$ and $95.6 \%$ for CEUS and $91.4 \%, 98.9 \%$ respectively, for MRI. When comparing the ROC curves, we found a higher area under curve for MRI $(0.952)$ then for CEUS $(0.835)(p=0.005)$, and $95 \%$ confidence interval of 0.0343 to 0.199 . No statistically significant difference in diagnosis of FLL was found between CEUS and MRI $(p>0.05)$ and the agreement between the two imaging techniques was good $(k=0.78)$. Conclusions: CEUS can be used as the first step in the diagnosis of liver lesions, but MRI remains the gold standard diagnostic method for liver tumors.
\end{abstract}

Keywords: contrast-enhanced ultrasound, magnetic resonance imaging, hepatocellular carcinoma, tumor grading

\section{Introduction}

Hepatocellular carcinoma (HCC) is one of the most common liver malignancies, frequently associated with cirrhosis [1]. Surveillance should be performed by experienced personnel in all at-risk populations using abdominal ultrasound every 6 months [1]. The enhancement pattern of the lesions is the key to the characterization of focal liver lesions (FLL), and in the estimation of the histological grade of malignancy [2,3]. Thus, computer tomography (CT), magnetic resonance imaging (MRI) or ultrasonography performed with intravenous contrast achieved a more accurate diagnosis of HCC in the new

Received 05.09.2013 Accepted 02.10.2013

Med Ultrason

2013, Vol. 15, No 4, 261-267

Corresponding author: Ioana Andreea Gheonea, MD, PhD, MSc

2, Petru Rares str

Craiova, Romania

Phone: +40751268732

E-mail: iagheonea@gmail.com guidelines [4]. Diagnosis should be based on imaging method with the identification of the typical hallmark of HCC: hypervascular in the arterial phase with washout in the portal venous or delayed phases [1]. Liver biopsy is recommended in uncertainly or inconclusive radiologic findings [1].

The aim of our study was to assess the role of transabdominal contrast enhanced US (CEUS) and MRI in characterization and detection of HCC in patients diagnosed with FLL.

\section{Material and methods}

A retrospective trial was conducted between January 2010-January 2013. A total number of 126 patients with focal liver lesions were evaluated in the Gastroenterology Department of Emergency Hospital Craiova, Research Center of Gastroenterology and Hepatology, University of Medicine and Pharmacy Craiova and SPAD Imaging. The final diagnosis was established by 
using clinical data, blood analysis, also imaging (CT/ MRI) and histopathological information. Final diagnosis of HCC was established in 76 patients evaluated by both imaging methods (MRI and CEUS). Patients with claustrophobic and metalic prothesis, recent myocardial infarction or severe respiratory inssuficiency were ruled out. Also, the patients following therapeutical procedures (ablation, embolization or antiangiogenic) were excluded. Histopathology was used only in 15 cases with initial non-conclusive results during imaging. The study was approved by the Local Ethical Committee. The technical equipment consisted of ultrasound systems with embedded contrast modules (Hitachi EUB 8500, Hitachi HI VISION Preirus) and a state-of-the-art MR scanner (Siemens MRI of 1.5 T). First of all, an US of the liver in the conventional mode, using the gray scale, was performed with a multifrequency convex array probe in order to identify the FLL. When the suspected lesion was identified, a dynamic CEUS was performed after the administration of SonoVue as a bolus, with the same convex array probe and the focus depth beyond the area of interest. Each patient received a $2.4 \mathrm{ml}$ i.v. bolus of contrast using a catheter placed in the ante-cubital vein, followed by $10 \mathrm{ml}$ of physiological saline flush. The enhancement patterns of the FLL were studied during the arterial (0-30 s), portal (30-120 s) and late phase up to 6 minutes, according to EFSUMB (European Federation of Socities for Ultrasound in Medicine and Biology) recommendations [1]. All images were digitally stored and analyzed by one experienced gastroenterologist. The lesions discovered on standard US were evaluated, if suspected for malignancy, by contrast enhanced MRI. MRI scans were performed using a 1.5-T MR imager. All patients underwent T1-weighted, T2-weighted, and Gd-DTPA enhanced (T1-weighted imaging with fat suppression) imaging with breath holding used as a breathing motion reduction technique. All patients received Gd-DTPA at a dose of $0.1 \mathrm{mmol} / \mathrm{kg}$ body weight. The lesions' signal intensities were categorized as hyperintense, isointense, and hypointense. MRI images were independently analyzed by one experienced radiologist. For each method of investigation we calculated the sensitivity, specificity, positive and negative predictive values (PPV and NPV), positive and negative likelihood ratio (+LR, $-\mathrm{LR})$, and accuracy, using a computer software (MedCalc Software 9, 2008, Mariakerke, Belgium), and we compare the ROC curves. We analyzed the significance of difference in diagnosing FLL by using the Chi-square test and a $p$ value $<0.05$ was considered to be statistically significant. Moreover, we assessed the agreement between the two imaging modalities by using the Kappa test. A good and very good agreement was considered when $\mathrm{k}$ values were between 0.60 and 1.00, and a poor, moderate, and fair agreement when $\mathrm{k}$ values were between 0.20 or smaller and 0.60 .

\section{Results}

We included in this study 126 patients with focal liver lesions, 75 men and 51 women, with a mean age of $59 \pm 10.56$ years. From these, 76 cases were diagnosed with HCC by using the two imaging methods (CEUS and MRI), with 69 patients having one lesion, 5 patients two lesions, and 2 patients multiple lesions, most patients being diagnosed in advanced stages. The clearest lesion on conventional ultrasound was selected for study in patients with more than one lesion. The mean size of the lesions was $5.2 \mathrm{~cm} \pm 3.3$ (range $1.2-15.1 \mathrm{~cm}$ ) in diameter. HCC appeared in context of viral B hepatitis in 2 cases, viral $\mathrm{C}$ hepatitis in 3 cases and cirrhosis in 30 cases. Sixty-six out of 126 cases were diagnosed as HCC by CEUS (fig 1, fig 2) and 73 out of 126 by MRI (fig 3, fig 4). The sensitivity, specificity, PPV, NPV, +LR, and -LR and accuracy for each method, in diagnosing $\mathrm{HCC}$, is shown

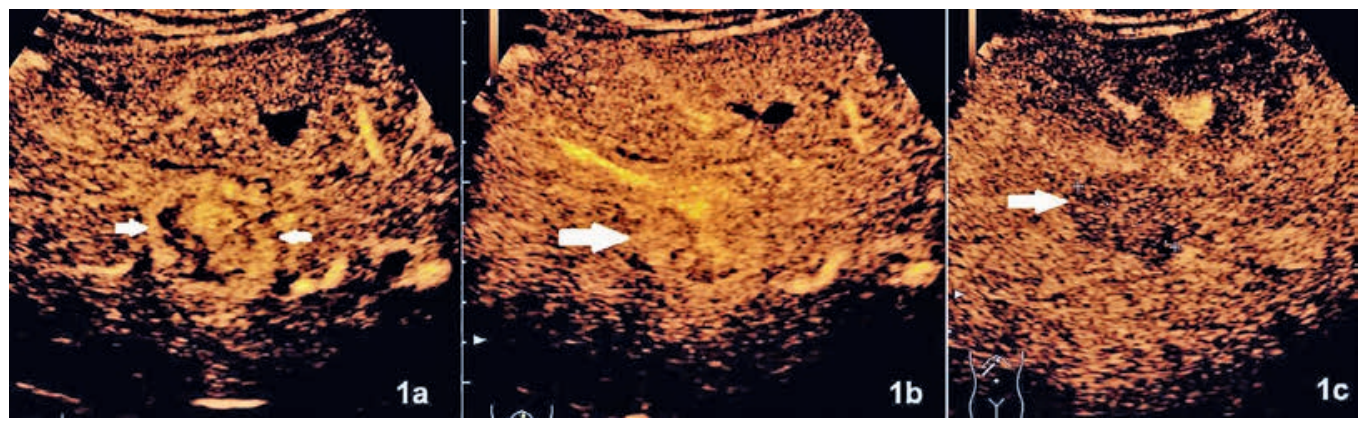

Fig 1. Patient with liver cirrhosis and one focal lesion in the right liver lobe. CEUS examination shows the nodule being quickly enhanced in the arterial phase, with a chaotic vascular pattern (a). In the portal venous phase the nodule is isoechoic (b) and show partial washout in the late phase (c). 
in Table I. When comparing the ROC curves, we found a higher area under the curve for MRI (0.952) than for CEUS (0.835), with a significantly $p$ value of 0.005 , and $95 \%$ confidence interval of 0.0343 to 0.199 (fig 5).

The 10 false negative cases diagnosed by CEUS did not show hyperenhancement in arterial phase or washout in the delayed phases and the 3 false negative cases diagnosed by MRI did not shown enhancement in arterial phase and were misdiagnosed by the two imaging techniques (moderately and well differentiated HCC). The seven cases misinterpreted by CEUS were correctly diagnosed by MRI. The four false positive cases classified by CEUS and one by MRI were histopathologically diagnosed as dysplastic nodules, focal nodular hyper-

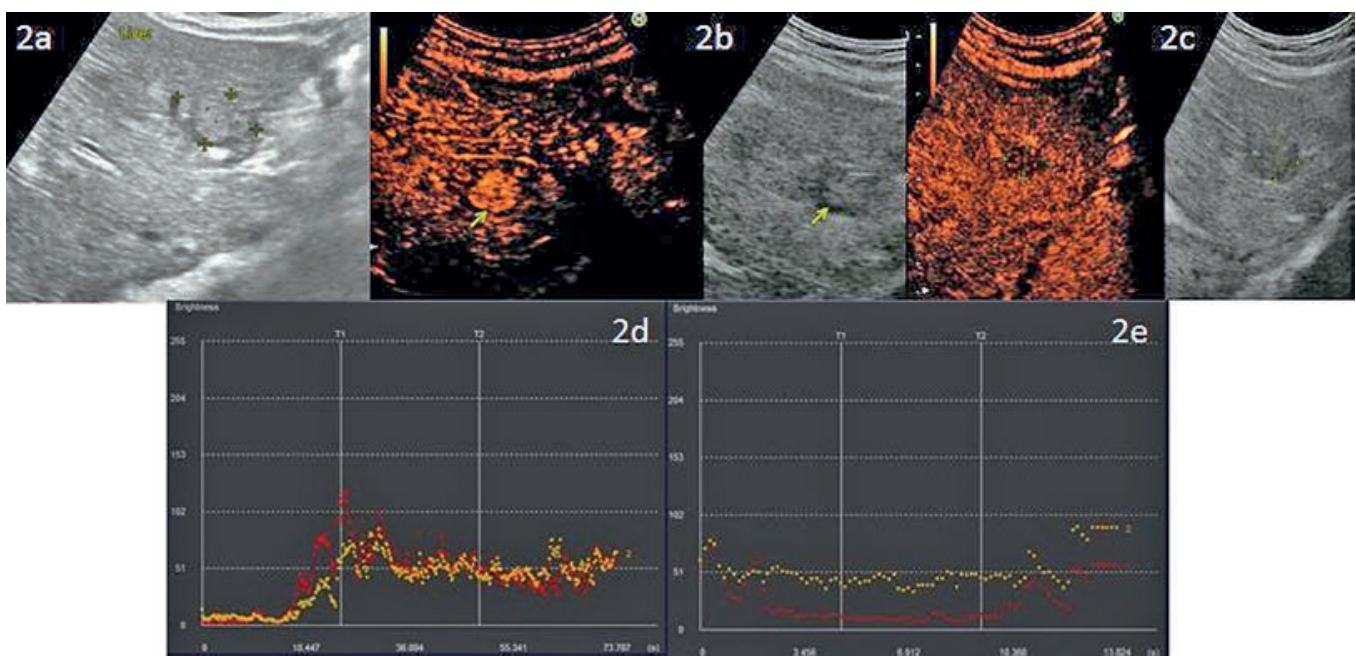

Fig 2. Patient with liver cirrhosis and a two cm nodule in the fifth segment (a). In CEUS the mass has the aspect of a hepatocarcinoma, hyperenhanced in the arterial phase (b), with partial washout in the late phase (c). Quantitative analysis of time intensity curves demonstrate the early and intensive uptake of the focal lesion (red curve) as compared to the liver parenchima (yellow curve) (d) and loss of contrast agent in the late phase compared to liver parenchima (e).

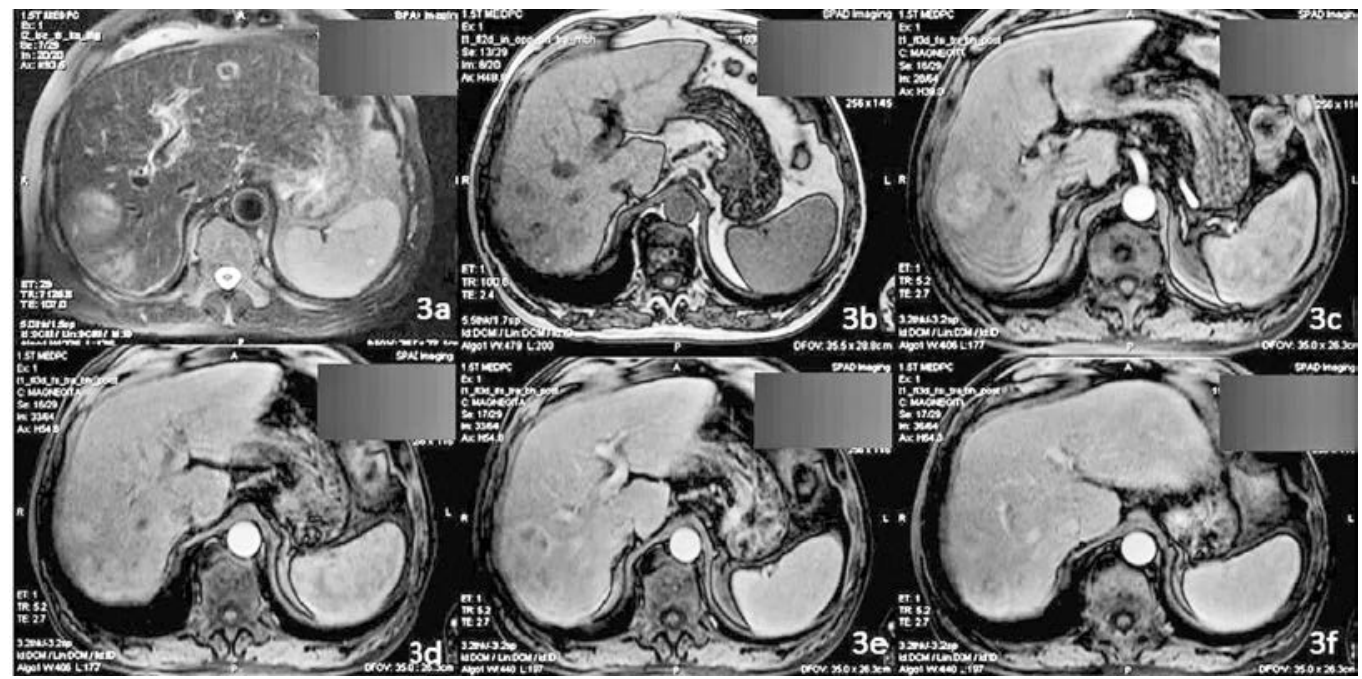

Fig 3. Hepatocellular carcinoma with different stages of differentiation. On T2-weighted images (a) a heterogeneously hyperintense lesion can be noted in the right live lobe. On the T1-weighted image (b) the lesion shows heterogenous hyposignal. The hypervascularity of the lesion and the presence of numerous nodules is depicted on arterial phase images after the bolus injection of Gd-BOPTA (c,d). The portal-venous phase image demonstrates contrast agent wash-out $(e, f)$. 


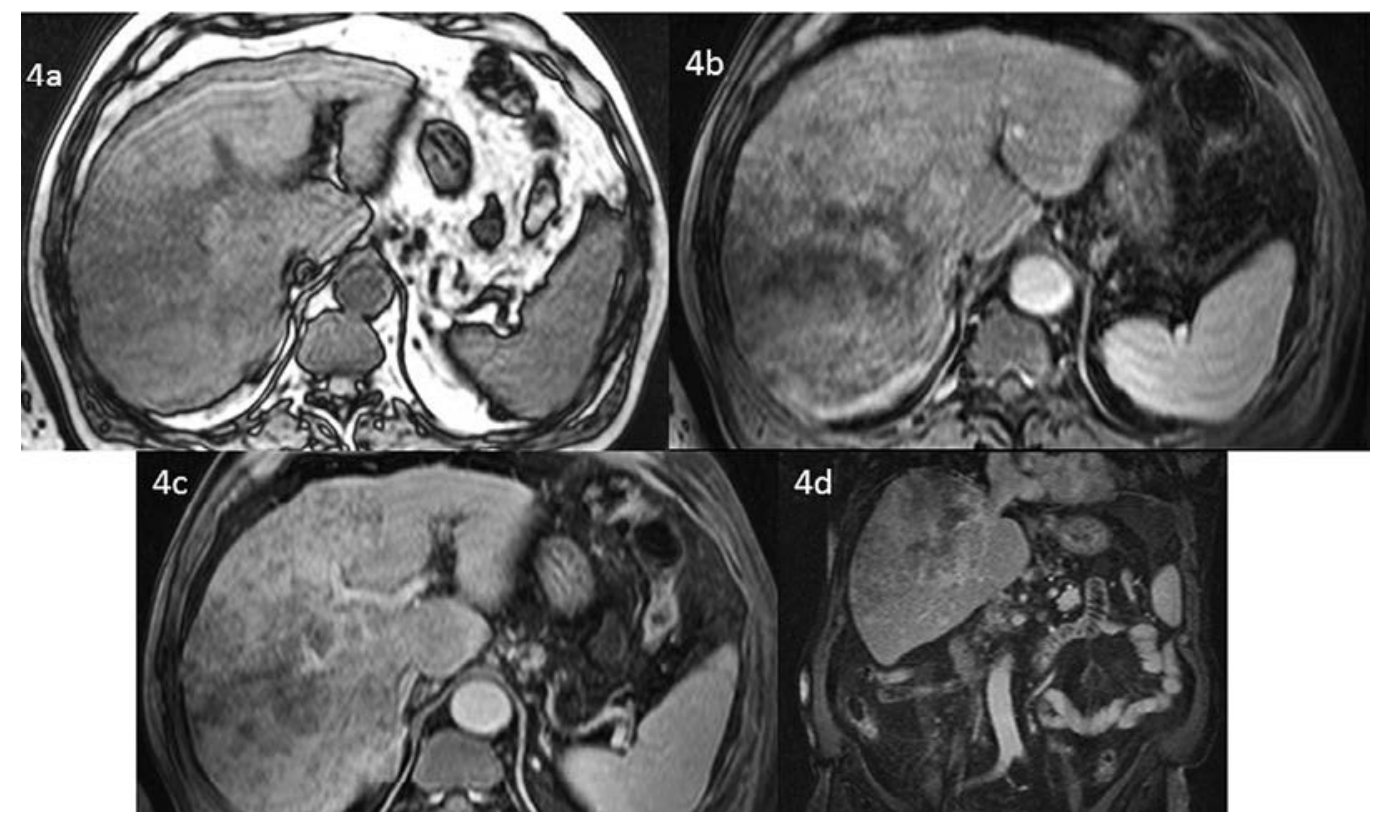

Fig 4. Diffuse poorly-differentiated hepatocellular carcinoma. On T1-weighted images the lesions appear hypointense (a). Arterial phase images after the injection of Gd-BOPTA (b) shows some hypervascular lesions. These lesions are hypointense compared to the normal liver in portal venous phase (c) and equilibrium phase (d).

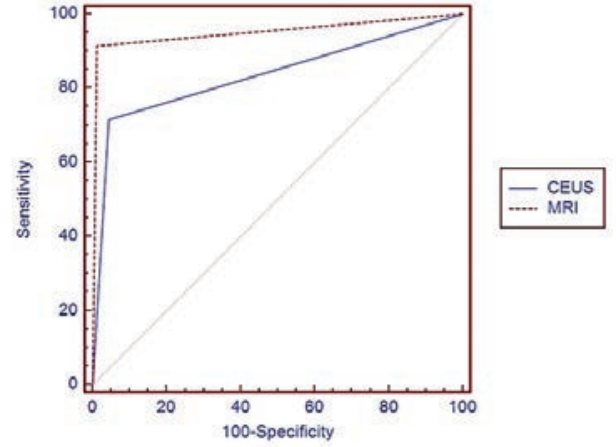

Fig 5. Comparing ROC curves for CEUS and MRI for the diagnosis of FLL.

Table I. The sensitivity, specificity, PPV, NPV, +LR, -LR and accuracy for CEUS and MRI

\begin{tabular}{lcc}
\hline Cases & $\begin{array}{c}\text { Imaging diagnostic techniques } \\
\text { CEUS }\end{array}$ & MRI \\
\hline Sensitivity & 71.4 & 91.4 \\
Specificity & 95.6 & 98.9 \\
PPV & 86.2 & 97 \\
NPV & 89.7 & 96.8 \\
+LR & 16.25 & 83.20 \\
-LR & 0.30 & 0.087 \\
Accuracy & 88.09 & 96.03 \\
\hline
\end{tabular}

plasia, cavernous hemangioma and hypervascular metastasis.

We did not found a statistically significant difference between CEUS and MRI in the characterization of FLL, while a good agreement between the two imaging modalities was observed with a $\mathrm{k}$ value of 0.78 .

\section{Discussion}

The characterization of liver lesions as benign or malignant is essential for the correct selection of patients to receive a good therapeutic management. With the newly introduced imaging technologies it is possible to diagnose $\mathrm{HCC}$ in early phases and to obtain cure, by using minimal invasive techniques $[1,5]$.

Several previous studies $[6,7]$ have demonstrated the high accuracy of CEUS in diagnosing and characterization of FLL. Developments in MRI technique and the availability of new MRI contrast agents have also improved liver lesion characterization [8]. These two imaging methods are based on the different enhancement patterns of liver lesions.

The recent introduction of microbbuble ultrasound contrast agents has had a major impact in diagnosing focal liver lesions. As a result of their double blood supply provided by the hepatic artery and portal vein, focal liver lesions present a complex temporal and spatial picture of 
increased and reduced contrast [9-11]. This new technique is based on digital processing of a signal produced by resonance of the microbubbles [11], while it enables the realtime visualisation of the enhancement pattern of lesions in all contrast phases [12]. The arterial phase provides information on the degree and type of tumor vascularity while the portal-venous and late phase differentiate between malignant and benign tumors. HCC are usually hyperenhancing in the arterial phase, typically with a chaotic vascular pattern. In the portal venous and late phase, HCC usually shows hypoenhancement apart from well-differentiated HCC that may be isoenhancing [13]. The typical CEUS pattern for HCC diagnosis, by using EFSUMB guide [13], is hyperenhancing in the arterial phase and hypoenhancing (slightly or moderately) in the late phase. In the recent studies this typical pattern corresponds to HCC in more than $97 \%$ of cases $[14,15]$. The CEUS performance in focal liver lesions diagnosis was assessed in many multicentric studies, the accuracy of method being between 85 and $90 \%$ [9, 16-18]. In two recent studies the accuracy was slightly lower ranging between 75 and $80 \%[16,18]$. Another study [19] showed that $90.3 \%$ of the nodules in patients with chronic liver diseases had a typical HCC arterial enhancement, but only $69.3 \%$ of them presented a washout in the late phase. Thus, if the typical HCC pattern is being considered (hyperenhancement in the arterial phase followed by washout in the portal and late phases) only $69.3 \%$ of cases were diagnosed by using CEUS.

In the present study, 10 of 76 lesions did not show hyperenhancement in the arterial phase or washout in the delayed phase, being thus false-negative. The sensitivity of CEUS in HCC diagnosis was $71.4 \%$, similar to other recent studies. The method is highly specific (95.6\%), the information obtained being also in accordance with the recent literature.

MRI plays an important role in assessment of liver lesions, being considered the gold standard imaging technique for HCC diagnosing. There are several studies which show a sensitivity of $92 \%$ [20] for double contrast enhanced MRI compared with $50 \%$ sensitivity in case of single contrast-enhanced MRI [21] in diagnosing nodules between 1-2 cm. In 2003, it has been demonstrated that angio-MRI increase the diagnostic accuracy of lesions less then $1 \mathrm{~cm}$ when comparing with $\mathrm{CT}$ (76\% versus $61 \%$ ), and particularly in case of lesions between 1 and $2 \mathrm{~cm}$ diameter ( $84 \%$ versus $47 \%$ ) [22]. In the present study, MRI correctly diagnosed the HCCs misdiagnosed by CEUS and a sensitivity of $91.4 \%$, with a specificity of $98.9 \%$ in HCC diagnosis has been found, regardless of nodules size. The various aspects of tumors in MRI depend on tumor architecture, grading, stromal component, as well as intracellular content [20]. The sensitivity of MRI in the detection of HCC is lower in cases with cirrhosis, in advanced stages and in the presence of ascites [23]. Patients with cirrhosis have foci of enhancement on arterial phase imaging that cannot be visualized on any other pulse sequences $[24,25]$. Difficulties can be observed in distinguishing well differentiated HCC from regenerative and dysplastic nodules, because small HCCs can have an identical appearance as dysplastic nodules, in up to $13 \%$ [26]. HCCs show variable enhancement with superparamagnetic iron oxide (SPIO) particles, therefore in the context of cirrhosis and small tumors, gadolinium enhanced imaging or a combination of the two types of contrast agent are preferred [27,28]. In our study, the incapacity of MRI to distinguish HCC depended on enhancement patterns and grading.

Recent studies have been focused on the relationship between cellular differentiation and imaging findings, such as contrast-enhanced CT (CECT), contrast-enhanced magnetic resonance imaging (CEMRI), Doppler ultrasound (DUS) and CEUS. Most of them proved that the tumor cellular differentiation was well correlated with imaging findings [29-31]; nevertheless this correlation has not been included in this study.

Regarding the comparison of ROC curves of the two imaging modalities, we found a higher sensitivity and specificity for contrast enhanced MRI in diagnosing HCC, which also concur with other published data. MRI had the advantage of diagnosing the small nodules and the vascular involvement. When using the Chi square test, no significant statistically difference $(\mathrm{p}>0.05)$ was observed between CEUS and MRI in characterization of focal liver lesions. Additionally, a good kappa value of 0.78 was observed between the two techniques.

In this study there are several limitations. First of all, it was not possible to use pathology as a reference in all FLL cases. Only fifteen cases were analyzed this way. Therefore, MRI was considered the standard in benign cases, especially when they had a typical vascular pattern such as FNH (focal nodular hyperplasia) or hemangiomas. Secondly, the HCC appeared in context of cirrhosis or other chronic liver disease [32]. HCC can have hypervascular enhancement in the arterial phase and isoenhancement in portal and delayed phases with the use of CEUS, thus overlapping with the appearance of FNH. Also, hypervascular metastases and HCC can have similar enhancement patterns, which might lead to false positive results [33].

\section{Conclusions}

In conclusion, the sensitivity and specificity of CEUS in diagnosing HCC was more then satisfactory, although 
MRI remains the most confident diagnostic tool for liver tumors. We think that CEUS can be used as the first step in the diagnosis of liver lesions, this imaging method having the advantage of safety, availability at the time of initial diagnosis, good tolerance by the patient, while being less expensive when compared with CT or MRI.

Acknowledgement: This work was supported by a grant of the Romanian National Authority for Scientific Research, Partnership project VIP SYSTEM, ID:20113,2-0503.

\section{Conflict of interest: none}

\section{References}

1. European Association For The Study Of The Liver; European Organisation For Research And Treatment Of Cancer. EASL-EORTC clinical practice guidelines: management of hepatocellular carcinoma. J Hepatol 2012; 56: 908-943.

2. Kim TK, Choi BI, Han JK, Hong HS, Park SH, Moon SG. Hepatic tumors: contrast agent-enhancement patterns with pulse - inversion harmonic US. Radiology 2000;216:411417.

3. Tanaka S, Ioka T, Oshikawa O, Hamada Y, Yoshioka F. Dynamic sonography of hepatic tumors. AJR Am J Roentgenol 2001;177:799-805.

4. Libbrecht L, Bielen D, Verslype Ch, et al. Focal lesions in cirrhotic explant livers: pathological evaluation and accuracy of pretransplantation imaging examinations. Liver Transpl 2002; 8: 749-761.

5. Gomaa AI, Khan SA, Leen EL, Waked I, Taylor-Robinson SD. Diagnosis of hepatocellular carcinoma. World J Gastroenterol 2009; 15: 1301-1314.

6. Lencioni R, Pinto F, Armillotta N, Bartolozzi C. Assessment of tumor vascularity in hepatocellular carcinoma: comparison of power Doppler US and color Doppler US. Radiology 1996; 201: 353-358.

7. Gaiani S, Casali A, Serra C, et al. Assessment of vascular patterns of small liver mass lesions: value and limitation of the different Doppler ultrasound modalities. Am J Gastroenterol 2000; 95: 3537-3546.

8. Ward J. New MR techniques for the detection of liver metastases. Cancer Imaging 2006; 6: 33-42.

9. Strobel D, Seitz K, Blank W, et al. Tumor-specific vascularization pattern of liver metastasis, hepatocellular carcinoma, hemangioma and focal nodular hyperplasia in the differential diagnosis of 1,349 liver lesions in contrast-enhanced ultrasound (CEUS). Ultraschall in Med 2009; 30: 376-382.

10. Forner A, Vilana R, Ayuso C, et al. Diagnosis of hepatic nodules $20 \mathrm{~mm}$ or smaller in cirrhosis: Prospective validation of the noninvasive diagnostic criteria for hepatocellular carcinoma. Hepatology 2008; 47: 97-104.
11. Wang Z, Tang J, An L, et al. Contrast-enhanced ultrasonography for assessment of tumor vascularity in hepatocellular carcinoma. J Ultrasound Med 2007; 26: 757-762.

12. Bolondi L, Gaiani S, Celli N, et al. Characterization of small nodules in cirrhosis by assessment of vascularity: the problem of hypovascular hepatocellular carcinoma. Hepatology 2005; 42: 27-35.

13. Claudon M, Dietrich CF, Choi BI, et al. Guidelines and good clinical practice recommendations for Contrast Enhanced Ultrasound (CEUS) in the liver - update 2012: A WFUMB-EFSUMB initiative in cooperation with representatives of AFSUMB, AIUM, ASUM, FLAUS and ICUS. Ultrasound Med Biol 2013; 39: 187-210.

14. Boozari B, Soudah B, Rifai K, et al. Grading of hypervascular hepatocellular carcinoma using late phase of contrast enhanced sonography - a prospective study. Dig Liver Dis 2011; 43: 484-490.

15. Fan ZH, Chen MH, Dai Y, et al. Evaluation of primary malignancies of the liver using contrast-enhanced sonography: correlation with pathology. AJR Am J Roentgenol 2006; 186: $1512-1519$.

16. Strobel D, Seitz K, Blank W, et al. Contrast-enhanced ultrasound for the characterization of focal liver lesions-diagnostic accuracy in clinical practice (DEGUM multicenter trial). Ultraschall Med 2008; 29: 499-505.

17. Tranquart F, Le Gouge A, Correas JM, et al. Role of contrast-enhanced ultrasound in the blinded assessment of focal lesions in comparison with MD-CT and CE-MRI: Results from a multicentre clinical trial. EJC 2008; suppl 6: 9-15.

18. Sporea I, Badea R, Martie A, et al. Contrast Enhanced U1trasound for the evaluation of focal liver lesions in daily practice. A multicentre study. Med Ultrason 2012; 14: 95100 .

19. Martie A, Sporea I, Sirli R, Popescu A, Danila M. How often hepatocellular carcinoma has a typical pattern in contrast enhanced ultrasound? Maedica (Buchar) 2012;7:236240.

20. Liu QY, Li HG, Gao M, Lin XF, Li Y, Chen JY. Primary clear cell carcinoma in the liver: CT and MRI findings. World J Gastroenterol 2011; 17: 946-952.

21. Fidler J, Hough D. Hepatocyte-specific magnetic resonance imaging contrast agents. Hepatology 2011; 53: 678-682.

22. Burrel M, Llovet JM, Ayuso C, et al. MRI angiography is superior to helical CT for detection of HCC prior to liver transplantation: an explant correlation. Hepatology 2003; 38: 1034-1042.

23. Teefey SA, Hildeboldt CC, Dehdashti F, et al. Detection of primary hepatic malignancy in liver transplant candidates: prospective comparison of CT, MR imaging, US, and PET. Radiology 2003; 226: 533-542.

24. Jeong YY, Yim NY, Kang HK. Hepatocellular carcinoma in the cirrhotic liver with helical CT and MRI: imaging spectrum and pitfalls of cirrhosis-related nodules. AJR Am JRoentgenol 2005; 185: 1024-1032.

25. Goshima S, Kanematsu M, Matsuo M, et al. Early-enhancing nonneoplastic lesions on gadolinium-enhanced magnet- 
ic resonance imaging of the liver following partial hepatectomy. J Magn Reson Imaging 2004; 20: 66-74.

26. Jeong YY, Mitchell DG, Kamishima T. Small $(<20 \mathrm{~mm})$ enhancing hepatic nodules seen on arterial phase MR imaging of the cirrhotic liver: clinical implications. AJR Am J Roentgenol 2002;178:1327-1334.

27. Semelka RC, Helmberger TK. Contrast agents for MR imaging of the liver. Radiology 2001; 218: 27-38.

28. Pauleit D, Textor J, Bachmann R, et al. Hepatocellular carcinoma: detection with gadolinium- and ferumoxidesenhanced MR imaging of the liver. Radiology 2002; 222: 73-80.

29. Kumada T, Nakano S, Toyoda H, et al. Assessment of tumor hemodynamics in small hepatocellular carcinoma: comparison of Doppler ultrasonography, angiography-assisted computed tomography, and pathological findings. Liver Int 2004; 24: 425-431.
30. Sakabe K, Yamamoto T, Kubo S, et al. Correlation between dynamic computed tomographic and histopathological findings in the diagnosis of small hepatocellular carcinoma. Dig Surg 2004; 21: 413-420.

31. Amano S, Ebara M, Yajima T, et al. Assessment of cancer cell differentiation in small hepatocellular carcinoma by computed tomography and magnetic resonance imaging. J Gastroenterol Hepatol 2003; 18: 273-279.

32. Giorgio A, Ferraioli G, Tarantino L, et al Contrast-enhanced sonographic appearance of hepatocellular carcinoma in patients with cirrhosis: comparison with contrast-enhanced helical CT appearance. AJR Am J Roentgenol 2004; 183: 1319-1326.

33. Sporea I, Sirli R, Martie A, Popescu A, Danila M. How useful is contrast enhanced ultrasonography for the characterization of focal liver lesions? J Gastrointestin Liver Dis 2010; 19: 393-398. 